\title{
THE ORIGIN OF BRIGHT X-RAY SOURCES IN MULTIPLE STARS
}

\author{
V. V. MaKarov ${ }^{1}$ and P. P. EgGleton ${ }^{2}$ \\ ${ }^{1}$ NASA Exoplanet Science Institute, California Institute of Technology, Pasadena, CA 91125, USA; valeri.makarov@jpl.nasa.gov \\ ${ }^{2}$ Lawrence Livermore National Laboratory, Livermore, CA 94550, USA; eggleton1@1lnl.gov \\ Received 2009 April 2; accepted 2009 August 18; published 2009 September 11
}

\begin{abstract}
Luminous X-ray stars are very often found in visual double or multiple stars. Binaries with periods of a few days possess the highest degree of coronal X-ray activity among regular, non-relativistic stars because of their fast, tidally driven rotation. But the orbital periods in visual double stars are too large for any direct interaction between the companions to take place. We suggest that most of the strongest X-ray components in resolved binaries are yetundiscovered short-period binaries, and that a few are merged remnants of such binaries. The omnipresence of shortperiod active stars, e.g., of BY-Dra-type binaries, in multiple systems is explained via the dynamical evolution of triple stars with large mutual inclinations. The dynamical perturbation on the inner pair pumps up the eccentricity in a cyclic manner, a phenomenon known as Kozai cycling. At times of close periapsis, tidal friction reduces the angular momentum of the binary, causing it to shrink. When the orbital period of the inner pair drops to a few days, fast surface rotation of the companions is driven by tidal forces, boosting activity by a few orders of magnitude. If the period drops still further, a merger may take place leaving a rapidly rotating active dwarf with only a distant companion.
\end{abstract}

Key words: binaries: close - stars: activity - stars: individual (AB Dor, BO Mic, TZ CrB)

Online-only material: color figures

\section{INTRODUCTION}

An intriguing relation has been found between binarity and X-ray activity of normal stars. For example, Makarov (2003) showed that of the 100 brightest X-ray sources within $50 \mathrm{pc}$ of the Sun, a high proportion (79) are in binaries. RS CVn-type spectroscopic binaries are by far the strongest X-ray sources among normal stars. These binaries comprise at least one evolved (luminosity class IV or III) companion and have orbital periods typically less than 10 days. Most of such objects have $\mathrm{X}$-ray luminosities in excess of $L_{X}=2.5 \cdot 10^{30} \mathrm{erg} \mathrm{s}^{-1}$. The origin of this enhanced coronal X-ray radiation is commonly accepted to lie in the high surface rotation rates supported by the orbital momentum and tidal friction (Biermann \& Hall 1976).

The graph in Figure 1 shows X-ray luminosities of all solartype ( $B-V \geqslant 0.4 \mathrm{mag}$ ) spectroscopic binaries with distances less than $25 \mathrm{pc}$ and periods less than 200 days listed in the SB9 catalog (Pourbaix et al. 2004), versus orbital periods in days. Objects not detected by ROSAT are marked with downward arrows. This set includes binaries of RS CVn type as well as main sequence binaries of BY-Dra type. Although the scatter is significant and the sample is small, the graph indicates that $L_{X}$ is correlated with the orbital period, and hence, with the rotation period in late-type spectroscopic binaries. The highest degrees of X-ray activity are found at periods between 1 and 10 days. A similar relation between $\log L_{X}$ and rotation period was derived for late dwarfs by Pizzolato et al. (2003; their Figure 3). As explained in Section 2, a brightness-limited survey of X-ray sources, such as ROSAT, is dominated by the most powerful emitters, those with $\log L_{X}$ well in excess of 29. In the Hyades open cluster, such sources mostly reside in spectroscopic binaries with orbital periods between 1 and 10 days (Stern et al. 1995). RS CVn-type binaries, which include at least one evolved component, are significantly brighter in $\mathrm{X}$-rays than dwarf binaries, and the peak of their $L_{X}$ distribution seems to be closer to 10 days (Makarov 2003). Singh et al. (1996) found that about $45 \%$ of field RS CVn-type objects they studied had $\log L_{X}>31$, but only $15 \%$ of Algol-type (semidetached) binaries were as bright. One of the possible explanations for this fact is the enhancement of X-rays by the mechanism of reconnection of magnetic lines around the detached RS $\mathrm{CVn}$ components, which are usually active. Interestingly, their Figure 4(a) suggests that $L_{X}$ goes down at periods less than 1 day, for these types of binaries, but Figure 4(b) indicates that the surface flux rises up to the shortest periods. Contact binaries of W UMa type are the shortest-period systems, whose X-ray luminosities are mostly between $\log L_{X}=29$ and 30 (McGale et al. 1996), noticeably weaker than the brightest BY and RS CVn-type emitters (Dempsey et al. 1993). These stars would occupy the area to the left and below the parabola's maximum in our Figure 1.

The tendency of strong X-ray radiation to appear in shortperiod (and therefore, rapidly rotating) binary systems is well documented and reasonably well understood. In this paper, we are concerned with the other part of the puzzle, namely, why these enhanced emitters are often found in wide multiple systems. Indeed, 20 out of a total 29 systems (67\%) are parts of hierarchical multiple systems in Figure 1, indicated with filled squares. The difference in $L_{X}$ distribution between single and binary $\mathrm{dK}$ dwarfs with orbital periods longer than $1 \mathrm{yr}$ in the Hyades is very pronounced, reaching almost an order of magnitude (Pye et al. 1994). Half of the high-fidelity Hyades members with $\log L_{X} / \log L_{\mathrm{Bol}}>-4.8$ reside in visual binaries with separations precluding any tidal interactions between the components (Makarov 2000). These results imply that enhanced $\mathrm{X}$-ray activity in normal stars occurs in short-period binaries or rapidly rotating stars, which, in their turn, mostly appear in multiple systems.

\section{X-RAY SOURCES IN VISUAL BINARIES}

There is a strong tendency for the brightest X-ray sources detected by the ROSAT All-Sky Survey to reside in resolved visual double stars (Makarov 2002). This would not be surprising if 


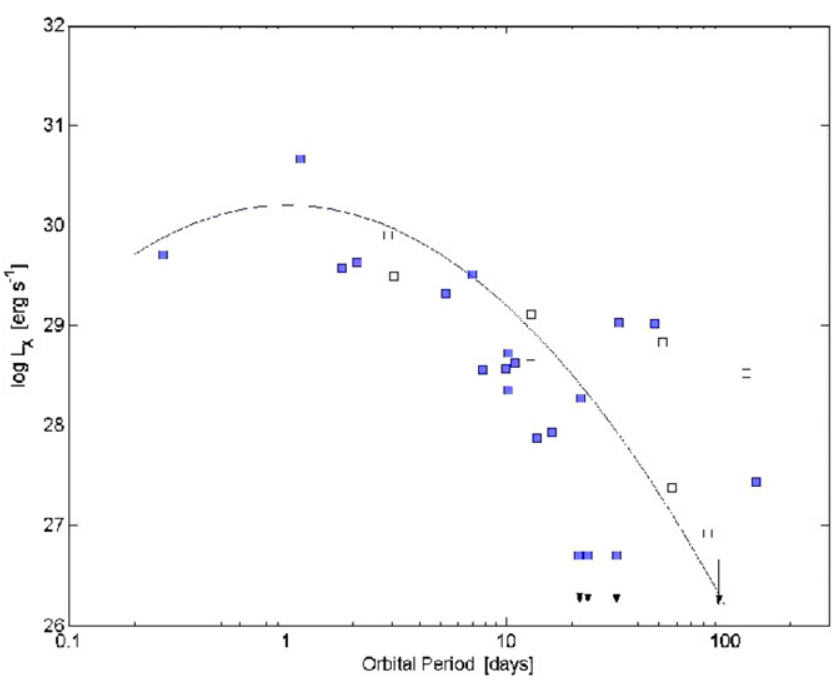

Figure 1. X-ray luminosities of all short-period solar-type spectroscopic binaries within $25 \mathrm{pc}$ of the Sun. A polynomial fit is shown only for reference. Binaries with known tertiary or multiple companions are marked with filled squares.

(A color version of this figure is available in the online journal.)

one could extend the previous argument about the short-period binaries to this case. However, many of these double stars are quite wide: they are visual or astrometric binaries, and there seems no obvious reason why these should be preferred. Components in wide binaries can be expected to be rapidly rotating if they are young, because young stars are usually rapidly rotating, but we would not expect their frequency in wide binaries to be higher than average. Besides, some of the prominent X-ray stars are known to be sufficiently old, such as the hard cataclysmic variable CQ Dra $\left(\log L_{X}=30.23, \mathrm{HR}=1\right)$ orbiting an $\mathrm{M}$ giant with a period of 1703 days (Reimers et al. 1988).

The answer comes from a closer examination of the most prodigious X-ray emitters among the nearby short-period binaries. Most of the binaries represented in Figure 1 are parts of wider, hierarchical multiple systems, as indicated with filled squares in this graph. If we limit ourselves to considering only binaries with periods less than 30 days, 17 out of 21 binaries $(81 \%)$ belong to multiple systems, i.e., have wider companions. Although the sample is small, the result is statistically strong. The active binary TZ CrB (HR 6063, HIP 79607) is a typical example of a very active, short-period ( $P=1.14$ days $)$, circularized binary in the solar vicinity. It is composed of two nearly identical solar analogs (masses 1.10 and $1.09 M_{\odot}$ ), but it also has a wider companion of similar mass $(P=889 \mathrm{yr}$, mass $1.06 M_{\odot}$ ) according to Tokovinin et al. (2006). The inner pair is a bright X-ray source with a count rate of 9.49 counts per second as measured by ROSAT. The tertiary companion is separated by 5.9 on the sky, and the distance to the system is approximately 20 pc. A similar triple star at $d=200$ pc would appear as a visual double with a separation of 0.6 , which could easily be resolved either in Hipparcos (resolution limit 0.'1; ESA 1997) or Tycho Double Star Catalog (TDSC; resolution limit 0.'3; Fabricius et al. 2002), the two large catalogs used by Makarov (2002) to identify double stars. The brightness of this system would be 0.095 counts per second, well above the sensitivity limit of the ROSAT Bright Source Catalog (RASS-BSC), 0.05 counts per second (Voges et al. 1999). Even at a distance of $400 \mathrm{pc}$, such a system would probably be detected in the ROSAT Faint Source Catalog (RASS-FSC; Voges et al. 2000), and still be resolved by Hipparcos as a visual double star. This example leads us to suspect that many of the double stars overluminous in X-rays are in fact yet-undiscovered triple systems with shortperiod inner binaries.

Assuming a typical hardness ratio for solar-type coronal sources of $\mathrm{HR} 1=-0.2$, we estimate that one-day binaries at $L_{X} \approx 10^{30} \mathrm{erg} \mathrm{s}^{-1}$ would be detected and listed in RASS$\mathrm{BSC}$ out to $150 \mathrm{pc}$. The detection radius for a 10-day binary at $L_{X} \approx 10^{29} \mathrm{erg} \mathrm{s}^{-1}$ would be approximately $50 \mathrm{pc}$. Evolved binaries of RS CVn type are considerably more luminous in X-rays than dwarfs, and can often be detected out to 300-400 pc. Therefore, an X-ray brightness-limited sample of nearby stars will comprise a large fraction of short-period binaries. The remaining difficulty is to explain why these binaries prefer to be accompanied by wider tertiary companions of similar mass.

Recently, Eggleton \& Kisseleva-Eggleton (2006, hereafter EKE06) suggested that AB Dor, one of the samples in Makarov (2003), had a history that involved (1) an initial multiplicity higher than two, and (2) a recent merger of two components of a close binary that now no longer exists except as a single merged remnant. In fact, AB Dor is the brightest component, visually as well as in X-rays, of a quadruple system, but for present purposes we disregard the distant companion Rst 137B (AB Dor B), which is itself a 0.'07 binary (Janson et al. 2007) at $\sim 9^{\prime \prime}$ from the principal component AB Dor A (Innis et al. 1986), and so we consider only the astrometric sub-binary AB Dor AC, with a period of $11.74 \mathrm{yr}$ (Nielsen et al. 2005). AB Dor $A$ is a very active and rapidly rotating $\mathrm{K}$ dwarf (0.51 days; Innis et al. 1988), and its X-ray brightness and high level of activity are no doubt due to this rapid rotation. $\mathrm{AB}$ Dor $\mathrm{C}$ is a very late (M7-M9) dwarf of $0.09 M_{\odot}$, near the very bottom of the hydrogen-burning main sequence (MS); it is sufficiently near the MS that it cannot be extremely young. Nielsen et al. (2005) estimated the age as $70 \pm 30$ Myr. It is not clear how $\mathrm{AB}$ Dor A, rotating near to its breakup rate, and subject to the mass ejection and the magnetic braking that is consequent on that spin, has maintained such extremely rapid rotation while most other stars of comparable age have braked considerably.

EKE06 suggested a prior evolution of $\mathrm{AB}$ Dor $\mathrm{AC}$ of the following form. The $11.74 \mathrm{yr}$ binary was once a triple system, but a close pair within this triple was driven, rather recently, into a merger. The present $\mathrm{AB}$ Dor $\mathrm{A}$ is the rapidly rotating remnant of that merger. If the merger took place only perhaps 1-5 Myr ago, we can understand why it is still rotating at close to breakup. The previous, now merged, close binary might have had an orbital period at birth of several days, or even weeks or months, and would have been driven into a merger by a combination of Kozai cycling and tidal friction (KCTF; Mazeh \& Shaham 1979; Kiseleva et al. 1998) that was dictated by AB Dor C, the companion in the 11.74 yr binary. Magnetic braking with tidal friction (MBTF; Huang 1966; Mestel 1968; van't Veer 1976; Rucinski 1983) probably also will have played a part in driving this sub-sub-binary to a merger.

The ultrafast rotator Speedy Mic (BO Mic, HIP 102626) is another example of an active isolated star in the solar neighborhood whose origin remains a mystery. This early $\mathrm{K}$ dwarf with a rotational period of 0.38 days (Cutispoto et al. 1997) and $\log L_{X}=31.07$ is not represented in Figure 1 because there is no documented evidence, to our knowledge, of a spectroscopic binarity. It has been known, however, since the publication of the Hipparcos catalog, as an astrometric binary with changing proper motion (ESA 1997). The invisible companion may be responsible for the outstanding properties of BO Mic, if it caused the original inner pair to merge recently, similar to the scenario discussed above for AB Dor. The orbital 
period of the companion is of particular interest, because $\mathrm{BO}$ Mic is suspected to be quite young, possibly a post-T Tauri star. If the orbital period is long, our surmise is refuted because the KCTF did not have enough time to merge the inner pair. We have attempted unconstrained orbital solutions based on the Hipparcos Intermediate Abscissae Data, using the method of Goldin \& Makarov (2006). In this case again, we encountered the persistent problem of unconstrained astrometric solutions on undersampled data, that the orbital eccentricity cannot be determined to the same relative precision as, for example, the period. Two alternative solutions were therefore produced, one with unconstrained eccentricity, and the other with eccentricity set to zero. The former solution is period $P=1146$ days, inclination $i=78^{\circ}$, eccentricity $e=0.63$, apparent semimajor axis $a_{0}=24.0$ mas, and parallax $\Pi=25.0$ mas. The latter solution is $P=1213$ days, $i=82^{\circ}, e=0.00, a_{0}=18.9$ mas, and $\Pi=23.1$ mas. Either of these solutions implies that the companion's mass is not much smaller than the primary, because the apparent orbital motion is slightly less than $1 \mathrm{AU}$, and the relative semimajor axis is slightly larger than $2 \mathrm{AU}$. The orbital period is short enough to make the KCTF merging scenario plausible.

\section{KOZAI CYCLES, TIDAL FRICTION, AND MAGNETIC BRAKING}

The essential points about Kozai cycling, tidal friction, and magnetic braking, are as follows.

1. If the inner orbit (with period $P_{\text {in }}$ ) of a triple is inclined at an angle $\eta$ to the outer orbit (period $P_{\text {out }}$ ), and if $\sin \eta \geqslant \sqrt{2 / 5}$ $\left(39^{\circ}<\eta<141^{\circ}\right)$, then the inner orbit is forced to cycle in eccentricity and in inclination, but not in period or semimajor axis, on a Kozai-cycling period $P_{\mathrm{KC}}$; see point (3) below. The amplitude of the eccentricity cycle depends only on (i) the minimum eccentricity and (ii) the inclination $\eta$. It does not depend on either the mass of the distant third body or its distance (i.e., on $P_{\text {out }}$ ). The range as a function of inclination and minimum eccentricity has been tabulated by Eggleton (2006; his Table 4.9). For instance, if $\eta=60^{\circ}$ the eccentricity can range between zero and 0.764 , or between 0.5 and 0.863 . An inclination of $60^{\circ}$ is the median to be expected if outer and inner orbits are oriented at random.

2. However, purest Kozai cycling requires that gravity be exactly inverse-square law. Modifications of gravity due to (i) general relativity (GR), (ii) the quadrupole moment of an extended star that rotates, and (iii) the quadrupole moments of each component due to the attraction of the other, can all destroy Kozai cycles, if these modifications are large enough. Very loosely, if $P_{\text {out }}(\mathrm{yr}) \gtrsim\left[P_{\text {in }}(\text { days })\right]^{1.4}$, the effect of Kozai cycling is suppressed. It is of course also suppressed at an opposite extreme: if $P_{\text {out }}(\mathrm{yr}) / P_{\text {in }}(\mathrm{yr}) \lesssim 4$, the inner orbit is likely to be so large relative to the outer orbit that the system is destroyed dynamically within a short time. For example, with $P_{\text {in }} \sim 16$ days, Kozai cycling can take place if $0.2 \mathrm{yr} \lesssim P_{\text {out }} \lesssim 50 \mathrm{yr}$.

3. The duration of a Kozai cycle is roughly $P_{\mathrm{KC}} \sim P_{\text {out }}^{2} / P_{\text {in }}$. $\left(m_{1}+m_{2}+m_{3}\right) / m_{3}$. Thus Kozai cycling can take place on timescales as short as a few centuries, but more typically on timescales of kiloyears to megayears, and even gigayears. With $P_{\text {in }} \sim 16$ days, $P_{\text {out }} \sim 12 \mathrm{yr}$ and $m_{3} \sim 0.1\left(m_{1}+m_{2}\right)$, the Kozai-cycle period is of order $3 \times 10^{4} \mathrm{yr}$.

4. To a good degree of approximation, the outer orbit is not affected at all by Kozai cycling.
5. Because the eccentricity $e_{\text {in }}$ can become large during a cycle while the semimajor axis $a_{\text {in }}$ is constant, the periastron separation $p \equiv a_{\text {in }}\left(1-e_{\text {in }}\right)$ can become small, and as a result tidal friction can become an important dissipative agent. The dissipation of energy reduces $a_{\text {in }}$ and $P_{\text {in }}$, just as in normal close but eccentric binaries. But in a purely binary situation the decrease of $a_{\text {in }}$ is limited by the fact that the orbit becomes circular and so dissipation stops: the final $a_{\text {in }}$ is roughly the initial semi-latus rectum. In the triple situation, there is no such limitation, and so we might expect $P_{\text {in }}$ to decrease indefinitely. However, this is limited by (2) above, so that the final product has a circular inner binary that no longer Kozai cycles, but is of sufficiently short period that either (i) the two components distort each other significantly, or (ii) their spins, in corotation with their orbit, cause enough of a quadrupole moment to suppress the Kozai cycling. In principle, the apsidal motion of GR might also do this, but in practice the other two effects are more important at short $P_{\text {in }}$.

6. The timescale $t_{\mathrm{TF}}$ of tidal friction can be estimated (Zahn 1977) as $t_{\mathrm{TF}} \sim t_{\mathrm{visc}}(p / R)^{8}$, where $R$ is the radius of the larger star; $t_{\text {visc }}$ is a viscous timescale inherent in the structure of the star, and is $\sim 1 \mathrm{yr}$. The fact that most binaries with periods $\lesssim 4 \mathrm{~d}$ have small or zero eccentricity is seen as confirmation that the circularization timescale is of the order of the nuclear timescale when the orbital radius is about ten times the stellar radius. In some old clusters, and the Galactic halo, orbits with periods as long as 10 days or even longer may be circularized (Latham et al. 1992).

7. If the components of the close pair are of spectral type $\mathrm{F}-\mathrm{M}$, and if its period becomes short ( $\lesssim 10$ days) as a result of KCTF, then orbital shrinkage will continue under the influence of magnetic braking, also in association with tidal friction-MBTF. F/G/K/M stars that rotate in $P_{\text {rot }} \lesssim$ 10 days are usually very $\mathrm{X}$-ray active (Figure 1 ), and this is normally attributed to dynamo activity driven by the combination of relatively rapid rotation and a turbulent surface convective zone. The dynamo activity means both that a stellar wind is driven off by the dissipation of a magnetic field as it emerges just outside the stellar surface, and that this wind carries off further the magnetic field. The magnetic field causes the wind to corotate with the star out to an Alfvén radius of several stellar radii (about $10 R_{\odot}$ in the case of the Sun itself), and thus carries off a great deal of angular momentum which, beyond the Alfvén radius, is lost to the star. In a single star, this would simply cause the star to spin more slowly, but in a binary whose components are close enough to be locked in corotation with the orbit by tidal friction this would cause the orbit to lose angular momentum and thus to spin faster. It is therefore an unstable process, although even as it runs away, with the period decreasing until the larger star is in contact with its Roche lobe at $\sim 0.3$ days (for the Sun), the timescale is unlikely to be much smaller than about $10 \mathrm{Myr}$.

8. KCTF can spin the close sub-binary up to $P_{\text {in }} \lesssim 3$ days, and then MBTF can spin it up further, by way of a very close but detached binary to Roche lobe overflow (RLOF). This can lead first to a semidetached "reverse Algol," where the loser is still the more massive component, and then probably either (i) to a normal (but very short-period) Algol or (ii) to a contact binary. Contact binaries are likely to evolve by net mass transfer from the less to the more massive component, until the less massive component is merged 

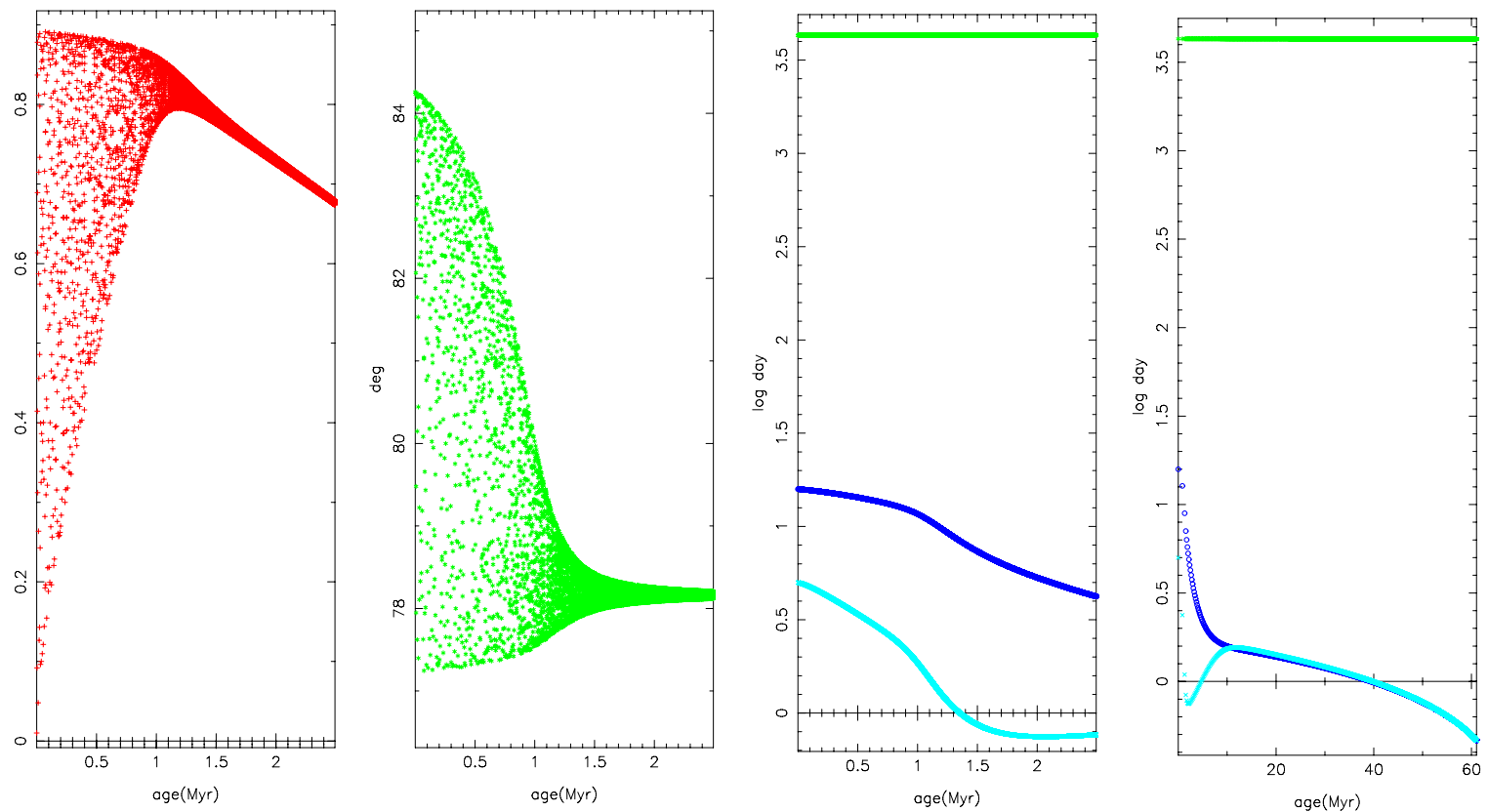

Figure 2. Behavior of eccentricity, inclination, and period for the inner binary of a triple proto-AB-Dor system. Left-hand panel: eccentricity as a function of time. The periodic (Kozai cycling) behavior at early times is severely undersampled. Second panel: mutual inclination as a function of time. Third panel: $P_{\text {in }}$, dark blue; $P_{\text {rot }}$, pale blue; $P_{\text {out }}$, green. See the text for details. Right-hand panel: as third panel, but on a longer timescale, so that the effect of MBTF leading to a merger at very short period is now visible.

(A color version of this figure is available in the online journal.)

into its companion (Webbink 1976). Such a process is likely to be quite slow, however, since contact binaries are by no means uncommon. But a further possibility (iii) is a rapid merger, on something like a timescale of years, and at its peak, days. Such a merger is to be expected if the mass ratio at the onset of RLOF is fairly extreme, like 2:1, 3:1, or even more; only if the mass ratio is rather mild, say $\lesssim 1.5: 1$, there is likely to be a settling down to a relatively longlived semidetached or contact state as in (i) or (ii). These evolutionary possibilities were discussed at some length by Yakut \& Eggleton (2005).

We illustrate the effect of KCTF and MBTF with an example that might be relevant to the $\mathrm{AB}$ Dor $\mathrm{AC}$ system. Consider a triple whose initial parameters, in an obvious notation, are

$$
\begin{aligned}
& \left(\left(\mathrm{K} 5 \mathrm{~V}+\mathrm{M} 7 \mathrm{~V} ; 0.65+0.15 M_{\odot} ; P_{\text {in }}=16 \mathrm{~d}, e_{\text {in }}=0\right)\right. \\
& \left.+\mathrm{M} 8 \mathrm{~V} ; 0.8+0.09 M_{\odot} ; P_{\text {out }}=11.74 \mathrm{yr}, e=0.61 ; \eta=84.3\right) .
\end{aligned}
$$

Since $\cos \eta=0.1$, the probability is $10 \%$ that the inclination will be thus large or larger, if the inclination is selected randomly because of some random collision of two primordial binaries. The initial rotational period of both components in the inner pair was set arbitrarily at 5 days. We computed the subsequent evolution according to the prescriptions of Eggleton (2006) for KCTF and MBTF.

The first two panels of Figure 2 show the evolution of the eccentricity and the inclination over an interval of about 2.5 Myr. In the first $0.1 \mathrm{Myr}$, the eccentricity (first panel) cycles over a considerable range, but the cyclic character of its variation is not easy to see on the scale plotted. The evolution is severely undersampled so that the variation of $e_{\text {in }}$ looks random. Tidal friction reduces the range of variation of $e_{\mathrm{in}}$, until after $\sim 1.5 \mathrm{Myr}$ the eccentricity fluctuates only slightly about a value of $\sim 0.75$. The inclination (second panel) also cycled originally, between about 77.3 and 84.3 , but settled down to a much narrower range about 78.2 by about 1.5 Myr. Subsequently, the eccentricity decreased while only fluctuating slightly, and halved itself roughly every $3 \mathrm{Myr}$. The third panel of Figure 2 shows the inner orbital period and the rotation period of the $\mathrm{K} 5 \mathrm{dwarf}$, for the first $2.5 \mathrm{Myr}$; and the fourth shows the same but for $\sim 60 \mathrm{Myr}$. KCTF is largely over after $\sim 10 \mathrm{Myr}$, with $P_{\text {in }}$ decreased to the value where the perturbative effects of (2) kill the cycling. But the period continues to decrease slowly because of MBTF, and then rather rapidly, presumably toward a merger, once $P \lesssim 0.5$ days at 60 Myr.

While the inner binary is still unmerged, it will be quite active (Figure 1) because its period is $\lesssim 10$ days. But after the merger, its rotational period will be $\sim 0.25$ days, and now increasing as a result of magnetic braking without tidal friction. The timescale for doubling the period will be about $20 \mathrm{Myr}$ - the code used did not simulate either the merger or the later evolution.

\section{DISCUSSION}

We interpret the abundance of wide double stars among the brightest X-ray sources as a direct consequence of the Kozai interaction in a hierarchical triple system, prerequisite to the generation of inner binaries with orbital periods less than 3 days. Can we find observational evidence in support of this hypothesis? A large-scale spectroscopic survey of randomly selected X-ray double stars would give a direct proof. Currently, only a few selected stars have been investigated in depth, for example, TZ CrB and AB Dor discussed in Section 2. But the main predictions of this model are supported by theory and a variety of astronomical observations.

1. The strongest X-ray sources among normal stars are binaries with a period of a few days.

2. Short-period binaries are mostly found in hierarchical triple or multiple systems. 
3. The timescales of tidal evolution and Kozai cycling in triple systems of high mutual inclination can be much shorter than the stellar evolution timescale.

4. Torres et al. (2003) examined 10 stars suspected to be members of the pre-MS TW Hya association (TWA). The candidate members were selected by high X-ray luminosities and kinematic or positional similarity to known TWA members. Despite this pre-selection, upon spectroscopic and radial velocity measurement none of the examined objects turned out to be young, but no less than six of them were confirmed as binaries. Even more strikingly, all binaries whose orbits could be determined had periods of less than 3 days. This result perhaps indicates that the overwhelming majority of bright X-ray sources in the field are short-period binaries, though most of them are yet to be discovered.

5. Tokovinin et al. (2006) looked at 165 known spectroscopic binaries with $P<30$ days. Some of these had already known third (or more) companions, but they also found 13 new ones by NACO adaptive optics. Making allowance by a maximum-likelihood algorithm for observational incompleteness, they found that the shortest-period spectroscopic binaries, those with $P<2.9$ days, were almost always (96\% likelihood) in triples, while the longest-period spectroscopic binaries, those with 12-30 days, were substantially less likely (34\%).

6. Pribulla \& Rucinski (2006) found that for all (88) known contact binaries in the northern sky down to $V=10,52$ (59\%) are in triples; Rucinski et al. (2007) increased that to $54(61 \%)$ with two new adaptive optics discoveries. This is a much higher proportion of triples than in a broad sample of binaries not confined to periods as short as in contact binaries $(<1$ day). Given the many selection effects that make the discovery of faint companions difficult, this result $(61 \%)$ is not inconsistent with $100 \%$ in practice.

7. Eggleton \& Tokovinin (2008) considered the multiplicities of the 4559 stellar systems brighter than the Hipparcos magnitude 6.0. They found that 1841 were at least binaries, and of these 404 were at least triples, i.e., about $22 \%$ of binaries have a further companion. However, among the 1841 that were at least binaries, the 89 that had a period $<3$ days contained $55(62 \%)$ that were at least triples.

8. Nuclear evolution may play a role in the evolution of some $\mathrm{X}$-ray sources, specifically the RS Cvn type. Although some of these have quite short periods, such as RS CVn itself (4.8 days), others have longer periods, e.g., RU Cnc (10.17 days) and RZ Eri (39.28 days). Both contain giants, and it is presumably the fact that the giant is fairly close to its Roche lobe, and forced by tidal friction to spin much more rapidly than it would have done if it had evolved as a single star, that makes it X-ray active. Probably KCTF and MBTF are irrelevant in these cases.

The above items seem consistent only with the concept that most, and arguably all, binaries with $P \lesssim 3$ days have a third body, and therefore that the third body must be essential for producing most or all of the short periods. This can only mean the KCTF mechanism; although MBTF can shorten periods from 5 or maybe 10 days to one day or less, there is no reason why this should involve a third body. Only one other mechanism might be worthy of consideration: within a cluster, gravitational scattering of hard binaries by other members of the cluster can harden the binaries, though generally by a rather small amount in each scattering. But once again there is no particular reason why the hardest binaries should end up as members of triples.
In summary, KCTF along with other physical processes can account for the observed properties of many X-ray sources, and perhaps all, or at least the great majority, with short periods. It can also account, through the merger mechanism described above specifically in the context of AB Dor, for X-ray sources in wide binaries. It is quite possible that luminous $\mathrm{X}$-ray sources in wide binaries are themselves in close binaries that have not yet been measured, and it is also possible that some are the merged remnants of close binaries. They need not be rotating as rapidly as $\mathrm{AB}$ Dor, which will probably be conspicuous for several hundred Myr yet until it has slowed down to perhaps $P_{\text {rot }} \sim 12$ days; but they could in principle be in wide binaries and have ages of several Gyr, having merged only after a substantially slower KCTF involving different initial conditions.

The research described in this paper was carried out partly at the Jet Propulsion Laboratory, California Institute of Technology, under a contract with the National Aeronautics and Space Administration. This work was also performed partly under the auspices of the U.S. Department of Energy by Lawrence Livermore National Laboratory under Contract DEAC52-07NA27344. This research has made use of the SIMBAD database, operated at CDS, Strasbourg, France; and data products from the 2MASS, which is a joint project of the University of Massachusetts and the Infrared Processing and Analysis Center, California Institute of Technology, funded by NASA and the NSF.

\section{REFERENCES}

Biermann, P., \& Hall, D. S. 1976, in IAU Symp. 73, Structure and Evolution of Close Binary Systems, ed. P. Eggleton, S. Mitton, \& J. Whelan (Dordrecht: Kluwer), 381

Cutispoto, G., Kürster, M., Pagano, M., \& Rodonò, M. 1997, IBVS, 4419 Dempsey, R. C., et al. 1993, ApJS, 86, 599

Eggleton, P. P. 2006, Evolutionary Processes in Binary and Multiple Stars (Cambridge: Cambridge Univ. Press)

Eggleton, P. P., \& Kisseleva-Eggleton, L. 2006, Ap\&SS, 304, 75 (EKE06)

Eggleton, P. P., \& Tokovinin, A. A. 2008, MNRAS, 389, 869

ESA 1997, The Hipparcos and Tycho Catalogues ESA SP-1200, Vols. 1-17

Fabricius, C., et al. 2002, A\&A, 384, 180

Goldin, A., \& Makarov, V. V. 2006, ApJS, 166, 341

Huang, S.-S. 1966, Ann. d'Astrophysique, 29, 331

Innis, J. L., Thompson, K., \& Coates, D. W. 1986, MNRAS, 223, 183

Innis, J. L., Thompson, K., Coates, D. W., \& Lloyd Evans, T. 1988, MNRAS, 235,1411

Janson, M., Brandner, W., Lenzen, R., Close, L., Nielsen, E., Hartung, M., Henning, T., \& Bouy, H. 2007, A\&A, 462, 615

Kiseleva, L. G., Eggleton, P. P., \& Mikkola, S. 1998, MNRAS, 300, 292

Latham, D. W., Mazeh, T., Stefanik, H. P., Davis, R. J., Carney, B. W., Torres, G., \& Laird, J. B. 1992, in ASP Conf. Ser. 32, IAU Coll. 135, Complementary Approaches to Double and Multiple Star Research, ed. H. A. McAlister \& W. I. Hartkopf (San Francisco, CA: ASP), 158

Makarov, V. V. 2000, A\&A, 358, L63

Makarov, V. V. 2002, ApJ, 576, L61

Makarov, V. V. 2003, AJ, 126, 1996

Mazeh, T., \& Shaham, J. 1979, A\&A, 77, 145

McGale, P. M., Pye, J. P., \& Hodgkin, S. T. 1996, MNRAS, 280, 627

Mestel, L. 1968, MNRAS, 138, 359

Nielsen, E. L., Close, L. M., Guirado, J. C., Biller, B. A., Lenzen, R., Brandner, W., Hartung, M., \& Lidman, C. 2005, Astron. Nachr., 326, 1033

Pizzolato, N., Maggio, A., Micela, G., Sciortino, S., \& Ventura, P. 2003, A\&A, 397, 147

Pourbaix, D., et al. 2004, A\&A, 424, 727

Pribulla, T., \& Rucinski, S. M. 2006, AJ, 131, 2986

Pye, J. P., et al. 1994, MNRAS, 266, 798

Reimers, D., et al. 1988, A\&A, 193, 180

Rucinski, S. M. 1983, Observatory, 103, 280

Rucinski, S. M., Pribulla, T., \& van Kerkwijk, M. H. 2007, AJ, 134, 2353

Singh, K. P., Drake, S. A., \& White, N. E. 1996, AJ, 111, 2415

Stern, R. A., Schmitt, J. H. M. M., \& Kahabka, P. T. 1995, ApJ, 448, 683 
Tokovinin, A., Thomas, S., Sterzik, M., \& Udry, S. 2006, A\&A, 450, 681

Torres, G., Guenther, E. W., Marschall, L. A., Neuhäuser, R., Latham, D. W., \& Stefanik, R. P. 2003, AJ, 125, 825

van't Veer, F. 1976, in IAU Symp. 73, Structure and Evolution of Close Binary Systems, ed. P. Eggleton, S. Mitton, \& J. Whelan (Dordrecht: Kluwer), 343
Voges, W., et al. 1999, A\&A, 349, 389, [ROSAT, RASS-BSC] Voges, W., et al. 2000, IAU Circ. 7432 [ROSAT RASS-FSC] Webbink, R. F. 1976, ApJ, 209, 829

Yakut, K., \& Eggleton, P. P. 2005, ApJ, 629, 1055

Zahn, J.-P. 1977, A\&A, 57, 383 